\title{
Comparación de la dureza superficial de diferentes tipos de materiales restauradores en premolares birradiculares, un estudio in vitro
}

\section{Comparison of surface hardness of different types of restoratives materials in premolars birradiculares, a in vitro study}

\author{
Taron Dunoyer A*, Frías Taron S**, Blanco Lerech S**, Camacho Vergara A***, \\ Bustillo JM****, Díaz Caballero $\mathrm{A}^{* * * * *}$
}

\section{RESUMEN}

Objetivo: Evaluar y comparar la dureza superficial en restauraciones con ionómero de vidrio y de resina compuesta en cavidades clase I según Black en premolares birradiculares.

Métodos: Se realizó un estudio descriptivo, comparativo in vitro donde se midió la dureza de dos tipos de materiales restauradores odontológicos, en cavidades clase I a dos distintas profundidades diseñadas en 32 premolares birradiculares. Distribuidos en 4 grupos con características distintas entre ellos, ya fuera por el material restaurador (ionómero de vidrio, resina compuesta) o por la profundidad de la cavidad (2-4 mm). Todas las muestras de cada grupo fueron sometidos a una fuerza vertical monitorizada a través del texturómetro EZ-S SHIMADZU hasta producir la fractura del material. Para evaluar la normalidad los datos obtenidos se sometieron a la prueba Shapiro Willk que rechazó la hipótesis nula. El análisis de los datos totales se realizó a través del test $t$ de Student para muestras independientes.

Resultados: Se evaluaron las durezas de la resina a 2 y $4 \mathrm{~mm}$ y el ionómero de vidrio a las mismas profundidades, encontrando una diferencia estadística a favor de la resina compuestas frente al ionómero de vidrio en ambas profundidades ( $p=0,000001445$ y $p=2,99 \mathrm{E}-9)$ y, en la comparación intragrupal, sólo diferencia significativa entre los dos grupos de resina a distinta profundidad $(p=0,016)$.

Conclusión: Los órganos dentarios con cavidades de $4 \mathrm{~mm}$ de profundidad que fueron restaurados con resina Tetric N-Ceram evaluados presentan mayor dureza que los restaurados con resina Vitremer a 2 y $4 \mathrm{~mm}$ y que la misma resina a $2 \mathrm{~mm}$ de profundidad.

Palabras clave: Resina, ionómero de vidrio, materiales de restauración, pruebas reológicas.

* Químico Farmacéutico. Universidad de Cartagena. Magíster en Biotecnología Universidad de la Habana. Candidato a Doctor en Ciencias de los Alimentos, Universidad de la Habana. Profesor Universidad de Cartagena.

** Odontólogo. Facultad de Odontología. Universidad de Cartagena.

*** Ingeniero de Alimentos. Universidad de Cartagena. Magíster en Ciencia y Tecnología de los Alimentos. Laboratorio de análisis físico químico. Universidad de Cartagena.

**** Odontólogo. Universidad de Cartagena. Especialista en Ortodoncia. Universidad de São Paulo. Candidato a Magíster en Estadística Aplicada. Universidad del Norte. Profesor Universidad de Cartagena.

***** Odontólogo. Universidad de Cartagena. Especialista en Periodoncia Universidad Javeriana. Magíster en Educación. Universidad del Norte. Doctor en Ciencias Biomédicas. Universidad de Cartagena. Profesor Universidad de Cartagena. 


\section{SUMMARY}

Objective: To evaluate and compare the surface hardness on restorations with glass ionomer and composite resin in Class I cavities according to Black in birradiculares premolars.

Methods: Descriptive, comparative In Vitro study, in which the hardness of two types of restorative materials was measured was performed in cavities class I at two different depths designed in 32 birradiculares premolars, which were divided into 4 groups with different including features, either by the restorative material (glass ionomer, composite resin) or the depth of the cavity $(2-4 \mathrm{~mm})$, all specimens from each group were subjected to a vertical force through the texturometer monitored to generate the fracture of the material. To assess normal data obtained were subjected to the test Shapiro Wilk to reject the null hypothesis, while the total data analysis was performed using Student's t-test for independent samples.

Results: Evaluated the hardness of the resin at 2 and $4 \mathrm{~mm}$ and the glass ionomer to the same depths, showing a statistical difference in favor of the composite resin Vs glass ionomer both depths $(p=0.000001445 p=2.99 \mathrm{E}-9)$ and intragroup comparison showing only significant difference between the two groups at different depths resin $(p=0.016)$. Conclusion: Of the materials tested in this comparative hardness study, the dental organs with cavities $4 \mathrm{~mm}$ depth were restored with Tetric $\mathrm{N}$-Ceram have greater hardness than Vitremer 2 and $4 \mathrm{~mm}$ and the same resin to $2 \mathrm{~mm}$ depth.

Key words: Resin, glass ionomer, restorative materials, rheological tests.

Fecha de recepción: 28 de enero de 2015.

Aceptado para publicación: 6 de abril de 2015.

Taron Dunoyer A, Frías Taron S, Blanco Lerech S, Camacho Vergara A, Bustillo JM, Díaz Caballero A. Comparación de la dureza superficial de diferentes tipos de materiales restauradores en premolares birradiculares, un estudio in vitro. Av. Odontoestomatol 2015; 31 (6): 355-361.

\section{INTRODUCCIÓN}

La caries es un proceso multifactorial degenerativo, que afecta los tejidos duros del diente, esmalte y dentina (1). Esta patología dental puede ser reversible en sus estadios tempranos como la mancha blanca o irreversible cuando ya hay presencia de cavitación. De ahí surge la necesidad en la odontología moderna de tener a la mano materiales de obturación idóneos y de posible mayor longevidad, para restaurar las cavidades que surgen a consecuencia de los procesos cariosos que tienen lugar en los dientes (2).

Diversas investigaciones demuestran que se desarrollan continuas mejoras en las propiedades de estos materiales $(3,4)$, sin embargo, aunque en menor magnitud se observan algunas deficiencias como: baja resistencia al desgaste, microfiltración, pigmentación y polimerización incompleta (5). La resistencia de estos materiales a diversos factores aún no es el ideal, dando como resultado su corta duración en boca (6).
Uno de los conceptos claves actuales es la odontología mínimamente invasiva, al momento de realizar las restauraciones dentales a los órganos que lo requieran (7), es que se debe ser lo más conservador posible con la estructura dental al momento de realizar la preparación necesaria, por tanto caen en desuso materiales que requieren preparaciones extensas para poder adquirir resistencia y adhesión con la estructura dental (8).

Existen ciertas características de los materiales que confieren seguridad al operador y permiten su empleo al momento de su elección, como lo son el coeficiente de expansión térmica, estabilidad del material, resistencia a las fuerzas de masticación, estética aceptable, dureza superficial, entre otras $(9,10)$. Para el desarrollo del presente estudio, se eligió la dureza superficial a la compresión de los materiales seleccionados.

El objetivo de este estudio fue comparar la dureza superficial, de la resina odontológica y el ionómero 
de vidrio en cavidades clase I de Black en dos diferentes espesores en un modelo de dientes previamente extraídos.

\section{MATERIALES Y MÉTODOS}

Se realizó un estudio descriptivo, comparativo in vitro, donde se evaluó la dureza superficial de un ionómero de vidrio reconstructor contra una resina compuesta nanohíbrida, utilizados para restaurar cavidades clase I de Black (11) con profundidades de 2 y $4 \mathrm{~mm}$ en premolares humanos. La muestra seleccionada por conveniencia, estuvo constituida por 32 premolares birradiculares, extraídos con fines ortodónticos y que no tuvieran anomalías del esmalte extensas.

La muestra se dividió en 2 grupos: el grupo A, para los dientes a los cuales se realizaron cavidades de 2 mm de profundidad y el grupo B, para los dientes a los cuales se les realizaron cavidades de $4 \mathrm{~mm}$ de profundidad. Se utilizó una fresa de diamante cilíndrica punta plana. A cada cavidad preparada se le rectificó la profundidad por medio de una sonda periodontal milimetrada de la casa comercial Hu-Friedy.

A cada uno de los dientes de la muestra se le confeccionó un soporte en acrílico de auto polimerizado para otorgarle estabilidad al ser posicionado en el instrumento de medición de la dureza superficial empleado.

Los dos grupos se dividieron en 2 subgrupos cada uno. El número 1 fue la denominación de los dientes utilizados como muestra restaurados con ionómero de vidrio reconstructor de la casa $3 \mathrm{M}$ : Vitremer ${ }^{\mathrm{TM}}$. El número 2, para los dientes restaurados con la resina nanohíbrida de la casa Ivoclar Vivadent: Tetric $\mathrm{N}$ Ceram.

- Grupo A1: Cavidades de $2 \mathrm{~mm}$ de profundidad obturadas con Vitremer ${ }^{\mathrm{TM}}$

- Grupo A2: Cavidades de $2 \mathrm{~mm}$ de profundidad obturadas con resina Tetric $\mathrm{N}$-Ceram

- Grupo B1: Cavidades de $4 \mathrm{~mm}$ de profundidad obturadas con Vitremer ${ }^{\mathrm{TM}}$

- Grupo B2: Cavidades de $4 \mathrm{~mm}$ de profundidad obturadas con resina Tetric N-Ceram

Todos los dientes de la muestra fueron sometidos a ensayos de esfuerzo con el texturómetro EZ-S SHIMADZU con número de serie 346-54909-33, de $50-60 \mathrm{~Hz}$ con un rango máximo de capacidad de 500 Newton, los dientes obturados y restaurados se sometieron a compresión en la cara oclusal con un área de contacto de $1 \mathrm{~mm}$ hasta lograr una profundidad de $1 \mathrm{~mm}$ en un solo avance. Monitorizando la fuerza necesaria para penetrar en sentido vertical la restauración existente en cada uno de los dientes de la muestra. Se resalta que la aplicación de la fuerza se realizó en el punto central de la restauración para todas las muestras de igual forma.

Las consideraciones éticas del proyecto estuvieron acorde a lo dispuesto en la resolución $n^{\circ} 008430$ de 1993 del Ministerio de Salud de la República de Colombia.

\section{Análisis estadístico}

Luego de la recolección de los datos se elaboró tabla matriz en Microsoft Excel versión para Windows 7.

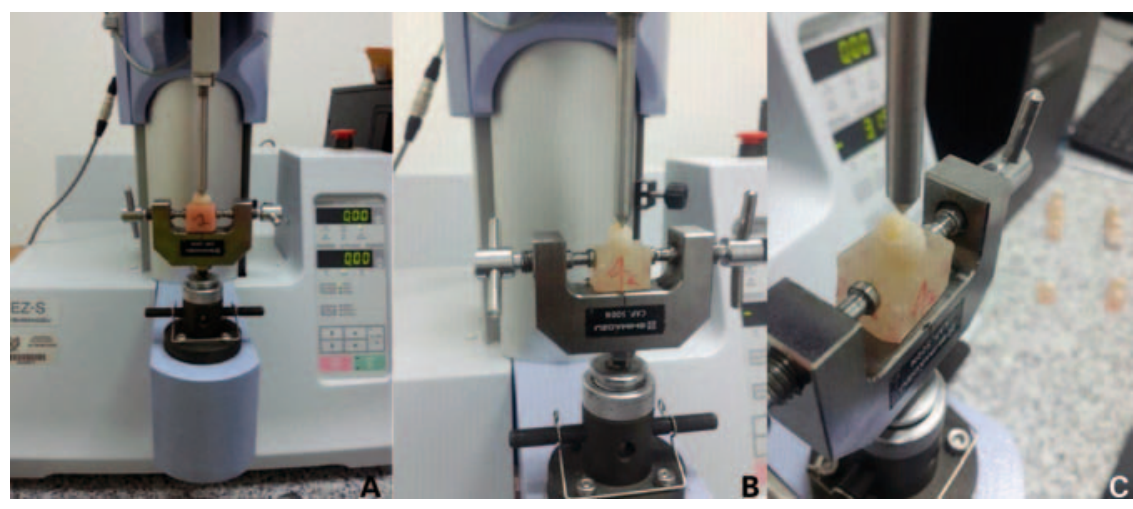

Fig. 1. Penetración superficial en dientes birradiculares obturados y restaurados a 2 y $4 \mathrm{~mm}$ de profundidad utilizando texturómetro EZ-S Shimadzu. A y B: Vista frontal de penetración. C: Vista lateral de penetración. 


\section{TABLA 1.- DATOS ESTADÍSTICOS DESCRIPTIVOS DEL GRUPO A1}

Grupo A1. Dientes con cavidades de $2 \mathrm{~mm}$ de profundidad obturadas con Vitremer ${ }^{\mathrm{TM}}$

\begin{tabular}{l|c} 
Media & 365,2484 \\
Mediana & 367,006 \\
Varianza & 175,918 \\
Desviación estándar & 13,263 \\
Rango intercuartil & 25,17 \\
Intervalo de confianza & $354,15-376,33$
\end{tabular}

\section{TABLA 2.- DATOS ESTADÍSTICOS DESCRIPTIVOS DEL GRUPO A2}

Grupo A2. Cavidades de $2 \mathrm{~mm}$ de profundidad obturadas con resina Tetric N-Ceram
Media

Mediana

Varianza

Desviación estándar

Rango intercuartil

Intervalo de confianza
420,6106

422,000

210,766

14,517

26,13

$408,47-432,74$
A continuación se aplicó la prueba de Shapiro Wilks la cual fue aplicada a cada una de las muestras obteniéndose los siguientes resultados: $A 1=0,448$; $\mathrm{A} 2=0,666 ; \mathrm{B} 1=0,992$ y B2=0,449. Como los valores son mayores a 0,05 no se puede rechazar la hipótesis de normalidad, esta prueba se efectuó mediante el programa SPSS stadistic v22 IBM. El análisis estadístico se realizó con la prueba t de muestras independientes con un nivel de significancia $p<0,05$ y la utilización del programa Statgraphics centurión XV.II.portable.

\section{RESULTADOS}

Aplicando la prueba $t$ para muestras independientes analizadas de dos en dos se obtuvieron los siguientes resultados.

Al comparar las cavidades de dos milímetros obturadas con Vitremer ${ }^{\mathrm{TM}}$ y la obturada con resina Tetric $\mathrm{N}$ -

\section{TABLA 3.- DATOS ESTADÍSTICOS DESCRIPTIVOS DEL GRUPO B1}

Grupo B1. Cavidades de $4 \mathrm{~mm}$ de profundidad obturadas con Vitremer ${ }^{\mathrm{TM}}$

\begin{tabular}{l|c}
\hline Media & 351,3664 \\
Mediana & 351,661 \\
Varianza & 228,118 \\
Desviación estándar & 15,103 \\
Rango intercuartil & 22,51 \\
Intervalo de confianza & $338,73-363,99$
\end{tabular}

\section{TABLA 4.- DATOS ESTADÍSTICOS DESCRIPTIVOS DEL GRUPO B2}

Grupo B2. Cavidades de $2 \mathrm{~mm}$ de profundidad obturadas con resina Tetric N-Ceram

\begin{tabular}{l|c}
\hline Media & 438,1161 \\
Mediana & 437,967 \\
Varianza & 122,504 \\
Desviación estándar & 11,068 \\
Rango intercuartil & 29,68 \\
Intervalo de confianza & $428,86-447,36$ \\
\hline
\end{tabular}

ceram, el resultado es que hay diferencia significativa $(p=0,000001445)$. Utilizando la prueba de Levene no se rechazó la hipótesis de igualdad de varianzas, por lo tanto las varianzas son homogéneas (sig. 0,0895). Alternativamente y apelando al intervalo de confianza, arroja el siguiente resultado: intervalo de confianza del $95 \%$ para la diferencia de medias suponiendo varianzas iguales $[-70,273,-40,450]$. Puesto que el intervalo de confianza no contiene el 0 , existe una diferencia estadísticamente significativa entre las medias de las dos muestras, con un nivel de confianza del 95\%. Siendo esta significancia mayor la de la resina por tener mayor media $(420,6106)$.

Cavidades de cuatro milímetros obturadas con Vitremer ${ }^{\mathrm{TM}}$ y resina Tetric $\mathrm{N}$-ceram el resultado es que hay diferencia significativa $(p=2,99 E-9)$. Prueba de Levene (sig. 0,500). El intervalo de confianza arrojó el siguiente resultado [-100,949,-72,5507]. Debido a que el intervalo de confianza no contiene el 0 , existe una diferencia estadísticamente significativa con un 
nivel de confianza del 95\%. Siendo mas significante la resina por tener mayor media $(438,1161)$. Observar las tablas 5 y 6 , comparación de dos muestras.

Al comparar las cavidades obturadas con Vitremer ${ }^{\mathrm{TM}}$ a dos y cuatro milímetros el resultado es que no hay significancia estadística $(p=0,07)$. Prueba de Levene (sig. 887). Intervalo de confianza [-1,332,29,124] Puesto que el intervalo contiene el 0 , no existe una diferencia estadísticamente significativa entre las dos muestras, con un nivel de confianza del $95 \%$.

En la comparación de las cavidades obturadas con resina Tetric N-ceram a dos y cuatro milímetros, el resultado es que hay diferencia significativa $(p=0,016)$. Prueba de Levene (sig. 0,621). Intervalo de confianza [-31,397,-3,6605]. Puesto que el intervalo no contiene el 0 , existe una diferencia estadísticamente significativa entre las medias de las dos muestras, con un nivel de confianza del 95\%. Siendo mayor la significancia de la resina a cuatro milímetros por tener mayor media $(438,1161)$.

\section{TABLA 5.- COMPARACIÓN DE RESULTADOS GRUPOS ENTRE RESINAS Y ENTRE IONÓMERO

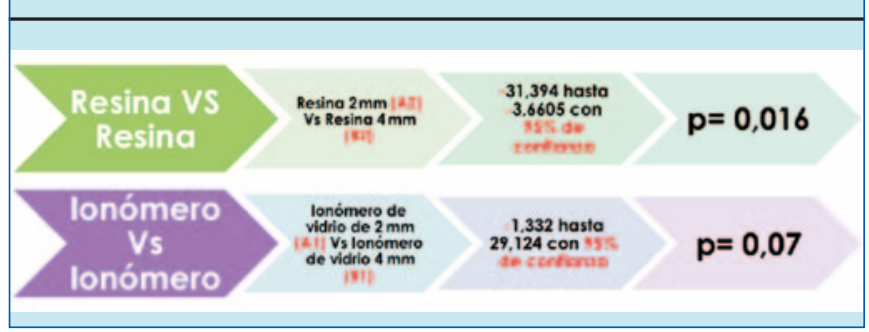

\section{TABLA 6.- COMPARACIÓN DE RESULTADOS GRUPOS ENTRE DIFERENTES MATERIALES A IGUALES PROFUNDIDADES}

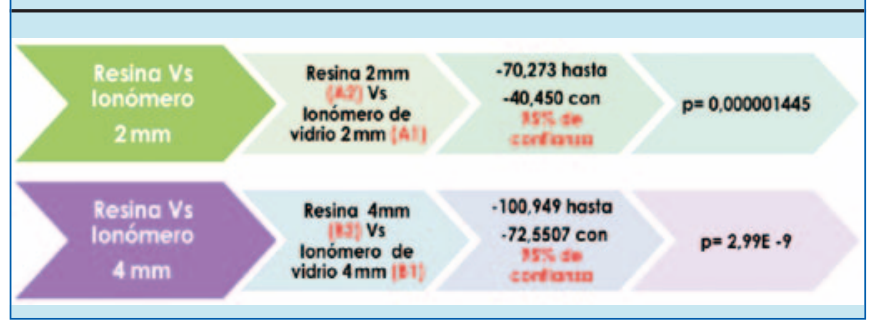

\section{DISCUSIÓN}

La selección del modelo para la aplicación de la evaluación de la dureza superficial que se escogió en el presente estudio, es diferente de los modelos aplicados por diversas investigaciones previas, tal como sucede con la investigación de Ozkan et al en 2013 (12), ellos trabajaron con discos de resinas como su modelo de trabajo, mientras que en la propuesta actual, el modelo propuesto y utilizado fue en cavidades preparadas en dientes previamente extraídos. Se considera que el modelo propuesto en la actual investigación, refleja una realidad mucho más aproximada a lo que sucede en la cavidad oral humana. Permitiendo, por lo tanto, una medición mucho más ajustada al contexto dental y odontológico. Situación similar al modelo de investigación propuesto por D'Alpino et al en 2011 (13), quienes trabajaron en terceros molares mandibulares extraídos evaluando la dureza de materiales de restauración con base de resinas.

El grosor de las resinas al parecer tiene una influencia en la calidad de la polimerización de ellas. Son et al, en 2014 (14), mencionan que diferentes marcas de resinas, tienen comportamientos de encogimiento y de dureza diferente al comparar diferentes marcas de resinas en sus investigaciones previas. Para evitar ese tipo de distorsiones en los datos obtenidos en el presente trabajo, se utilizó una sola marca de resina y en dos diferentes profundidades de 2 y de 4 $\mathrm{mm}$, lo que permite evaluar variaciones en la dureza superficial, evaluando con el texturómetro y no con el Vickers como herramienta de medición, tal como lo emplean ellos. De una forma similar con el presente trabajo, las profundidades con las que trabajaron fueron de 1, 2 y $3 \mathrm{~mm}$ en su modelo.

Thomé et al, en 2007 (15), evaluaron el efecto del grosor de los materiales activados con fotopolimerización, pero utilizan igualmente un modelo de resinas en discos, con un grosor de $5 \mathrm{~mm}$, grosor el cual nunca se alcanzó en las muestras empleadas en la presente investigación. Ya que se trabajó siempre con grosores de 2 y $4 \mathrm{~mm}$ en cada uno de los dientes de la muestra.

Como una posible estrategia de innovación, se debe mencionar que la utilización del texturómetro en la evaluación de la dureza superficial de dos diferentes 
materiales de obturación empleados en odontología, no tiene en la actualidad, muchas referencias de empleo de la mencionada herramienta de evaluación de la presente investigación. Ese enlace de tecnología y el empleo de un modelo de aplicación de fuerzas, con bastante similitud a la realidad oral, son factores a destacar.

La evaluación de la dureza superficial en diferentes materiales dentales, de una manera sistemática se emplean el test de dureza de Knoop (16) y el test de dureza de Vickers (17). Es de resaltar que en la presente investigación se decidió por el empleo del texturómetro EZ-D Shimadzu, ya que la principal diferencia con respecto a los otros test de dureza utilizados estriba en que los resultados obtenidos permiten tener una mejor correlación entre el comportamiento de la dureza de los materiales utilizados in vitro y el comportamiento de la resina y el ionómero en un ciclo masticatorio. A su vez este equipo simula de cierta forma los movimientos desarrollados en un proceso de masticación al aplicar una fuerza compresiva sobre estos materiales. Los test de dureza de Knoop y de Vickersofrece menos garantías de exactitud aunque se resalta su facilidad y la rapidez del ensayo y son recomendables para otros materiales restauradores con más dureza superficial tipo amalgamas (18).

Llevar a cabo estudios de evaluación de la dureza superficial de dos materiales de obturación de cavidades dentales en diferentes grosores o profundidades, es muy importante, ya que ayuda a refinar las indicaciones clínicas y la orientación a las fabricas de materiales dentales, buscando siempre un mayor beneficio para los pacientes odontológicos tal como lo resaltan Shanthala y Xavier en el 2013 (19). Factores estos que permite obtener tratamientos odontológicos mucho más duraderos y efectivos para los pacientes cuando se emplean ionómeros de vidrio o resinas como material de obturación.

\section{CONCLUSIONES}

Bajo las condiciones de este estudio in vitro, se puede concluir que la resina Tetric $\mathrm{N}$-Ceram presenta mayor dureza superficial al compararla con el ionómero de vidrio Vitremer ${ }^{\mathrm{TM}}$ independientemente de la profundidad de la cavidad, siendo esta diferencia estadísticamente significativa a los dos milímetros de profundidad $(p=0,000001445)$ y a los cuatro milímetros de profundidad ( $p=2,99 E-9)$. Sin embargo, es necesario realizar mas estudios de estos materiales para determinar si esta diferencia se comporta de la misma manera en condiciones in vivo.

Los órganos dentarios con cavidades de $4 \mathrm{~mm}$ de profundidad que fueron restaurados con resina Tetric $\mathrm{N}$-Ceram evaluados presentan mayor dureza que los restaurados con resina Vitremer a 2 y $4 \mathrm{~mm}$ y que la misma resina a $2 \mathrm{~mm}$ de profundidad.

La resina evaluada posee una dureza significativamente mayor a otros tipos de materiales que aún son de amplio uso tal como el ionómero de vidrio reconstructor, para restaurar órganos dentarios posteriores.

\section{BIBLIOGRAFÍA}

1. Kalesinskas P, Kacergius T, Ambrozaitis A, Peciuliene V, Ericson D, et al. Reducing dental plaque formation and caries development. A review of current methods and implications for novel pharmaceuticals. Stomatologija/issued by public institution "Odontologijos studija" 2014; 16(2):44-52.

2. Hosseinalipour M, Javadpour J, Rezaie H, Dadras T, Hayati AN. Investigation of mechanical properties of experimental Bis-GMA/TEGDMA dental composite resins containing various mass fractions of silica nanoparticles. Journal of prosthodontics: official journal of the American College of Prosthodontists. 2010;19(2):112-7.

3. Dong Q, Chow LC, Wang T, Frukhtbeyn SA, Wang $F$, Yang $M$, et al. A New Bioactive Polylactidebased Composite with High Mechanical Strength. Colloids and surfaces A, Physicochemical and engineering aspects. 2014;457:256-62.

4. Xu HH, Weir MD, Sun L. Calcium and phosphate ion releasing composite: effect of $\mathrm{pH}$ on release and mechanical properties. Dental materials: official publication of the Academy of Dental Materials. 2009;25(4):535-42. 
5. Xu HH, Moreau JL. Dental glass-reinforced composite for caries inhibition: calcium phosphate ion release and mechanical properties. Journal of biomedical materials research Part B, Applied biomaterials. 2010;92(2):332-40.

6. Hatai Y. Extreme masking: achieving predictable outcomes in challenging situations with lithium disilicate bonded restorations. The international journal of esthetic dentistry. 2014;9(2):206-22.

7. Mackenzie L, Banerjee A. The minimally invasive management of early occlusal caries: a practical guide. Primary dental journal. 2014;3(2):34-41.

8. Roulet JF, Geraldeli S. Adhesion is the key. The journal of adhesive dentistry. 2013;15(5):403.

9. Fareed MA, Stamboulis A. Nanoclay addition to a conventional glass ionomer cements: Influence on physical properties. European journal of dentistry. 2014;8(4):456-63.

10. Almeida-Chetti VA, Macchi RL, Iglesias ME. Effect of post-curing treatment on mechanical properties of composite resins. Acta odontologica latinoamericana: AOL. 2014;27(2):72-6.

11. Larson TD. Extension for prevention: margin placement. Northwest dentistry. 2012;91(2):126, 7, 9 passim.

12. Ozcan S, Yikilgan I, Uctasli MB, Bala O, Kurklu ZG. Comparison of time-dependent changes in the surface hardness of different composite resins. European journal of dentistry. 2013;7 (Suppl 1):S20-5.

13. D’Alpino PH, Bechtold J, dos Santos PJ, Alonso RC, Di Hipolito V, Silikas N, et al. Methacrylateand silorane-based composite restorations: hardness, depth of cure and interfacial gap formation as a function of the energy dose. Dental materials: official publication of the Academy of Dental Materials. 2011;27(11):1162-9.

14. Son SA, Roh HM, Hur B, Kwon YH, Park JK. The effect of resin thickness on polymerization characteristics of silorane-based composite resin.
Restorative dentistry $\mathcal{E}$ endodontics. 2014;39 (4):310-8.

15. Thome T, Steagall W, Jr., Tachibana A, Braga SR, Turbino ML. Influence of the distance of the curing light source and composite shade on hardness of two composites. Journal of applied oral science: revista FOB. 2007;15(6):486-91.

16. Torabi K, Rasaeipour S, Ghodsi S, Khaledi AA, Vojdani M. Evaluation of the Effect of a Home Bleaching Agent on Surface Characteristics of Indirect Esthetic Restorative Materials-Part II Microhardness. The journal of contemporary dental practice. $2014 ; 15(4): 438-43$.

17. Kaminedi RR, Penumatsa NV, Priya T, Baroudi K. The influence of finishing/polishing time and cooling system on surface roughness and microhardness of two different types of composite resin restorations. Journal of International Society of Preventive $\&$ Community Dentistry. 2014;4(Suppl 2):S99-S104.

18. Shaini FJ, Fleming GJ, Shortall AC, Marquis PM. A comparison of the mechanical properties of a gallium-based alloy with a spherical high-copper amalgam. Dental materials: official publication of the Academy of Dental Materials. 2001;17(2): 142-8.

19. Shanthala GS, Xavier MK. The effect of thermocycling on fracture toughness and hardness of different core build up materials. Indian journal of dental research: official publication of Indian Society for Dental Research. 2013;24(6):653-8.

\section{CORRESPONDENCIA}

Antonio Díaz

Grupo de investigaciones GITOUC

Facultad de Odontología.

Universidad de Cartagena

Campus de la salud

Cartagena, Colombia

E-mail: adiazc1@unicartagena.edu.co 